\title{
Dermatology
}

Dermatology 2009;218:178-180

DOI: $10.1159 / 000182270$

\section{Acute Acne Flare following Isotretinoin Administration: Potential Protective Role of Low Starting Dose}

\author{
A. Borghi, L. Mantovani, S. Minghetti, A. Virgili, V. Bettoli \\ Section of Dermatology, Department of Clinical and \\ Experimental Medicine, University of Ferrara, Ferrara, Italy
}

\section{Key Words}

Oral isotretinoin $\cdot$ Acne $\cdot$ Flare-up

Oral isotretinoin (13-cis-retinoic acid) is indicated to treat severe acne unresponsive to other therapies. It is the only drug currently available that affects each of the pathogenic factors involved in acne. This explains its near universal efficacy for the treatment of acne. Moreover, oral isotretinoin leads to remission that may be permanent.

The recommended daily dose is $0.5-1 \mathrm{mg} / \mathrm{kg}$ of body weight. Treatment of severe forms of acne should be continued until a cumulative dose of $120-150 \mathrm{mg} / \mathrm{kg}$ is reached as doses $<120 \mathrm{mg} / \mathrm{kg}$ are associated with increased rates of post-treatment relapses $[1$, 2]. Otherwise, total doses $>150 \mathrm{mg} / \mathrm{kg}$ are not of further therapeutic benefit.

Oral isotretinoin therapy is associated with numerous adverse effects, which are usually dose dependent $[3,4]$. Acute in- flammatory flares may occur during the first 3-5 weeks of treatment [5]. The flares manifest by paradoxical aggravation of acne, notably of the inflammatory component. As a rule, mild forms of flare resolve spontaneously and do not require change or discontinuation of treatment. Sometimes, inflammatory flare-up can be particularly severe and may distress the patient. These severe flares must be distinguished from acne fulminans, which is accompanied by systemic signs, articular symptoms and biochemical signs of inflammation [6]. Severe inflammatory acne deterioration may lead to scar development. In severe cases, systemic steroid therapy and either reduction or discontinuation of isotretinoin are necessary. The actual responsibility of oral isotretinoin in the extent of these flares is not proven. However, improvements usually occur upon discontinuation of isotretinoin, while increases in dosage can aggravate the inflammation. In order to avoid acne flares, many authors suggest to start oral isotretinoin treatment with a dose of no more than $0.5 \mathrm{mg} / \mathrm{kg} /$ day for the first month, and then to increase the daily dosage to $1 \mathrm{mg} / \mathrm{kg}$ [7].

With the present observational study, we wanted to assess whether initial daily doses $<0.5 \mathrm{mg} / \mathrm{kg}$ can further reduce the occurrence of flares during the first month of isotretinoin administration. Between October 2002 and October 2007, 132 acne patients (67 males and 65 females, mean age 20.3 years) whose clinical features are reported in table 1 , group 1 , were treated with oral isotretinoin at an initial daily dose $\leq 0.2 \mathrm{mg} / \mathrm{kg}$. The dosage was subsequently increased by $5 \mathrm{mg}$ every 2 weeks, until the maximum tolerated dose (between 0.5 and $1 \mathrm{mg} / \mathrm{kg}$ of body weight) was reached. All patients were treated until acne clearing, without exceeding a total cumulative dose of $150 \mathrm{mg} / \mathrm{kg}$. With the aim of

Table 1. Demographic and clinical features of acne patients treated with oral isotretinoin (OI)

\begin{tabular}{|c|c|c|c|c|c|c|c|}
\hline Patients & Males & Females & $\begin{array}{l}\text { Mean age, } \\
\text { years }\end{array}$ & $\begin{array}{l}\text { Mean acne } \\
\text { grade (face) }\end{array}$ & $\begin{array}{l}\text { Mean acne dura- } \\
\text { tion before OI, years }\end{array}$ & $\begin{array}{l}\text { Patients with } \\
\text { trunk involvement }\end{array}$ & Flare-up \\
\hline \multicolumn{8}{|c|}{ Group 1 (initial daily dose $\leq 0.2 \mathrm{mg} / \mathrm{kg}$ ) } \\
\hline 132 & $67(50.7)$ & $65(49.3)$ & 20.3 & 1.03 & 7.4 & $67(51)$ & $10(7.5)$ \\
\hline \multicolumn{8}{|c|}{ Group 2 (initial daily dose $0.5 \mathrm{mg} / \mathrm{kg}$ ) } \\
\hline 142 & $61(43)$ & $81(57)$ & 20.4 & 1.3 & 6.8 & $79(56)$ & $22(15.5)$ \\
\hline
\end{tabular}

Figures in parentheses are percentages. 
Table 2. Demographic and clinical features of patients developing acne flare during the first 4 weeks of oral isotretinoin (OI) treatment

\begin{tabular}{|c|c|c|c|c|c|c|c|c|c|}
\hline \multirow{2}{*}{$\begin{array}{l}\text { Patients } \\
\text { with flare }\end{array}$} & \multicolumn{2}{|c|}{ Flare } & \multirow[t]{2}{*}{ Males } & \multirow[t]{2}{*}{ Females } & \multirow{2}{*}{$\begin{array}{l}\text { Mean age, } \\
\text { years }\end{array}$} & \multirow{2}{*}{$\begin{array}{l}\text { Mean acne } \\
\text { grade (face) }\end{array}$} & \multirow{2}{*}{$\begin{array}{l}\text { Mean acne duration } \\
\text { before OI, years }\end{array}$} & \multirow{2}{*}{$\begin{array}{l}\text { Mean acne } \\
\text { worsening, \% }\end{array}$} & \multirow{2}{*}{$\begin{array}{l}\text { Patients with trunk } \\
\text { involvement }\end{array}$} \\
\hline & face & trunk & & & & & & & \\
\hline \multicolumn{10}{|c|}{ Group 1 (initial daily dose $\leq 0.2 \mathrm{mg} / \mathrm{kg}$ ) } \\
\hline $10(7.5)$ & 10 & 0 & $4(6)$ & $6(9)$ & 20 & 0.8 & 6.9 & 63 & $3(30)$ \\
\hline \multicolumn{10}{|c|}{ Group 2 (initial daily dose $0.5 \mathrm{mg} / \mathrm{kg}$ ) } \\
\hline $22(15.5)$ & 22 & 3 & $9(14.7)$ & $13(16)$ & 19.9 & 1.4 & 7.0 & 56 & $9(41)$ \\
\hline
\end{tabular}

Figures in parentheses are percentages.

finding a comparative population, acne patients treated with oral isotretinoin over a 4-year period (1998-2002) were retrospectively examined. Among these patients, 142 had received an initial dose of $0.5 \mathrm{mg} / \mathrm{kg} / \mathrm{day}$, increased weekly by $5 \mathrm{mg}$ up to the maximum tolerated dose, i.e. $\leq 1 \mathrm{mg} / \mathrm{kg}$ (table 1, group 2). Treatment was discontinued when acne remission was achieved, with a total cumulative dose $\leq 150 \mathrm{mg} / \mathrm{kg}$.

Demographic and disease characteristics were well matched between the two groups. It must be stressed that the dosing strategy was the sole difference in patient management between the two compared groups. Since the presence of multiple large closed comedones prior to treatment is considered the greatest risk factor for the development of flares [8], we cleared closed comedones with light cautery after applying a topical anesthetic before the administration of isotretinoin. The following data were assessed at baseline and monthly: (1) acne severity using the Leeds grading system [9] and lesion counting, (2) clinical side effects, (3) patient satisfaction with the outcome of treatment. For the entire patient population and across the periods, patients were always assessed by the same physician. Patients were asked to contact our department in case of self-assessed acne deterioration. If this was the case, a visit was scheduled to calculate acne grade and count lesions. Flare-up was defined as acne grade and/or lesion count $\geq 30 \%$ compared to baseline. Since a quantitative definition of flare is not available in the literature, this grade of aggravation was chosen arbitrarily as severe enough to usually require some medical interventions in our clinical practice.

As shown in table 2, 10 (7.5\%) and 22 patients (15.5\%) developed flares during the first 4 weeks of treatment in groups 1 and 2 , respectively $(\mathrm{p}=0.0415)$. Among patients with flares, all presented facial involvement. Flares of truncal acne were less common (table 2). No alterations in general health suggesting a diagnosis of acne fulminans were recorded in any patient. Institution of short-term systemic steroid therapy and reduction of isotretinoin provided rapid improvement of inflammation in all cases. In no patient was definitive suspension of oral isotretinoin necessary.

The findings reported here seem to suggest that an isotretinoin dosage regimen starting with a low dose $(\leq 0.2 \mathrm{mg} / \mathrm{kg})$ may reduce the occurrence of flares during the first 4 weeks of treatment. Gradually reaching a standard daily dose and a recommended total cumulative dose should provide immediate and long-term efficacy not different from therapies starting with higher doses. Consistent with this, several lines of evidence have shown that cumulative dosing is more important than daily dosing in the prevention of acne relapses $[10,11]$.

The role of the initial daily dose of isotretinoin in the development of acne fulminans is controversial as cases of acne fulminans have been reported with initial dosages $<0.5$ [5]. Nonetheless, experimental evidence of the correlation between initial dose of isotretinoin and a risk of developing acne fulminans is lacking. The responsibility of the starting dose of isotretinoin in inducing inflammatory flares after the first month of treatment has never been documented either.

The main limitation of the results reported herein is that they do not arise from a randomized and controlled study. However, to our knowledge, no previous studies have compared different isotretinoin dose regimens in large numbers of acne patients in order to assess differences in the occurrence of flares during the first weeks of treatment.

It could be argued that a protocol starting with a lower daily dose may represent a potential disadvantage for patients since it requires a longer course of treatment than a conventional dose regimen. Moreover, this regimen might seem more complex as it implies slow and gradual increases in the daily isotretinoin dosage. Taking this into account, patients should receive detailed counseling from the prescriber and a written schedule of daily isotretinoin intake prior to treatment. Needless to say that among our patient population no difficulties occurred in following the low-dose treatment protocol.

In conclusion, in our opinion, a dosage schedule potentially reducing the occurrence of acute acne flares and the related physical and psychological distress may enhance patients' satisfaction and compliance with treatment.

\section{References}

$\checkmark 1$ Layton A, Cunliffe WJ: Guidelines for optimal use of isotretinoin in acne. J Am Acad Dermatol 1992;27:S2-S7.

$\checkmark 2$ Katsambas AD, Stefanaki C, Cunliffe WJ: Guidelines for treating acne. Clin Dermatol 2004;22:439-444.

-3 Charakida A, Mouser PE, Chu AC: Safety and side effects of the acne drug, oral isotretinoin. Expert Opin Drug Saf 2004;3:119-129.

4 Lupi-Pégurier L, Muller-Bolla M, Fontas E, Ortonne JP: Reduced salivary flow induced by systemic isotretinoin may lead to dental decay. A prospective clinical study. Dermatology 2007;214:221-226. 
5 Chivot M: Acne flares and deteriorations during oral isotretinoin therapy. Ann Dermatol Venereol 2001;128:224-228.

$\checkmark 6$ Choi EH, Bang D: Acne fulminans and 13-cis-retinoic acid. J Dermatol 1992;19:378-383.

7 Lehucher Ceyrac D, Chaspoux C, Sulimovic L, Morel P, Lefrancq H: Aggravation de l'acné sous isotrétinoïne. 6 cas, facteurs prédictifs. Ann Dermatol Venereol 1998;125:496-499.

8 Bottomley WW, Cunliffe WJ: Severe flares of acne following isotretinoin: large closed comedones (macrocomedones) are a risk factor. Acta Derm Venereol 1993;73:74.

$\checkmark 9$ Burke BM, Cunliffe WJ: The assessment of acne vulgaris - the Leeds technique. Br J Dermatol 1984;111:83-92.
10 Harms M: Isotretinoin: 10 years on. Dermatology 1993;186:81-82.

11 Lehucher-Ceyrac D, Weber-Buisset M: Isotretinoin and acne in practice - a prospective analysis of 188 cases over 9 years. Dermatology 1993;186:123-128.

Annarosa Virgili

Section of Dermatology

Department of Clinical and Experimental Medicine

University of Ferrara, Via Savonarola 9

IT-44100 Ferrara (Italy)

Tel. +39 0532 205825, Fax +390532 206791, E-Mail vri@unife.it 